\title{
LOS GEOPARQUES COMO RECURSO PARA LA ENSEÑANZA-APRENDIZAJE DEL ESPACIO GEOGRÁFICO EN EDUCACIÓN PRIMARIA: EL PAISAJE DE LAS ÁREAS DE MONTAÑA
}

Rubén Fernández Álvarez ${ }^{1}$

Recibido: 10/01/2019

Aceptado: 19/09/2019

\section{RESUMEN:}

El desarrollo de la red Geoparques ha supuesto el impulso de un nuevo elemento didáctico. Esta figura pretende convertirse en el nexo de unión entre el trabajo en las aulas y el que se lleva a cabo en el campo. Todo ello supone la creación de una red educativa de carácter internacional en la que los geoparques y los centros educativos intercambien conocimientos y técnicas que repercutan de forma positiva en el proceso de enseñanza-aprendizaje de los paisajes de montaña.

\section{Palabras Clave:}

Didáctica de la Geografía; Paisaje; Geoparques; Educación Primaria; Convenio Europeo del Paisaje.

\section{Abstract:}

The development of the Geoparques network has led to the promotion of a new didactic element. This figure aims to become the connection between work in the classroom and

1 Departamento de Geografía. Universidad de Salamanca.rfa@usal.es 
work in the field. All this implies the creation of an international educational network in which Geoparks and educational centres exchange knowledge and techniques that have a positive impact on the teaching-learning process of mountain landscapes.

\section{KEYWORDS:}

Didactic of Geography; Landscape; Geoparks; Primary education; European Landscape Convention.

\section{RÉSUMÉ:}

Le développement du réseau Geoparques a conduit à la promotion d'un nouvel élément didactique. Le Géoparc vise à faire le lien entre le travail en classe et celui qui se déroule sur le terrain. Tout ceci implique la création d'un réseau éducatif international dans lequel les Géoparcs et les centres éducatifs échangeront des connaissances et des techniques qui ont un impact positif sur le processus d'enseignement-apprentissage des paysages de montagne.

\section{MoTS-CLÉS:}

Enseignement de la géographie ; Paysage; Éducation primaire; Convention européenne du paysage.

\section{INTRODUCCIÓN Y OBJETIVOS}

La elaboración del Convenio Europeo del Paisaje (CEP) en octubre del año 2000, determina un marco normativo de carácter internacional (Prats y Busquets, 2010; Zoido, 2000) que aporta unas líneas básicas de actuación en materia de paisaje. Entre otros aspectos, pone de relevancia el notable interés que suscita la formación desde la base educativa, pues considera que una ciudadanía formada en paisaje puede contribuir de forma destacada a su gestión y ordenación (Zoido, 2000). No es posible ordenar aquello que no se conoce, así, para poder gestionar adecuadamente el paisaje es necesario, además de conocer los elementos que lo integran, entender las interrelaciones que de ellos surgen y como repercuten en el espacio (Zoido, 2006a). Del mismo modo, el paisaje es la expresión visual del espacio geográfico, por ello su conocimiento ayudará a comprender parte de los procesos acaecidos en el territorio, así como su evolución (Miralbés e Higueras, 1993). Con el paisaje como instrumento didáctico es posible poner en marcha el proceso de enseñanza-aprendizaje del territorio y de los elementos que en este intervienen (Batllorí y Serra, 2017). Asimismo, para su estudio, se han de tener en cuenta las cualidades perceptivas y sociales que emanan del paisaje, pues ellas influirán en la forma en que los estudiantes lo interpretarán y lo sentirán (Martínez y Arrebola, 2016). 
Desde el CEP se propone la siguiente definición: "por paisaje se entenderá cualquier parte del territorio tal como la percibe la población, cuyo carácter sea el resultado de la acción y la interacción de factores naturales y/o humanos" (Consejo de Europa, 2000: 1). Nos encontramos ante una definición de carácter integrador (Zoido, 2000; Mata, 2006; Serrano, 2007; Oliva et al., 2014) en la que se pone de manifiesto que se considera paisaje a cualquier parte del territorio independientemente de sus cualidades, situando al mismo nivel la vertiente natural y antrópica (Mata, 2004; Busquets, 2010). Este puede ser un notable punto de partida para enfrentarse al proceso de enseñanza del paisaje, pues con ella, adaptada al nivel formativo adecuado, podría acometerse la explicación del término paisaje y, sobre todo, la importancia que en su configuración tiene la acción humana (Nardi, 2010). Igualmente, a partir del CEP podría extraerse la no adjetivación del paisaje (Zoido, 2000), es decir, no hay que valorar a un paisaje por sus condiciones estéticas, más bien por lo que puede suponer ese paisaje y por su carácter identitario (Hernández, 2009). Es decir, el tratamiento se ha de realizar en función, también, de las percepciones que de este medio tiene sus habitantes y no de la belleza del mismo (Busquets, 2010). Desde el ámbito educativo el paisaje se ha de entender como un instrumento cuyas posibilidades didácticas aumentan con su tratamiento en las aulas y en el trabajo de campo (Bajo, 2001). La enseñanza de la geografía se apoya frecuentemente en el trabajo de campo (Busquets, 2010; Cardona, 2013; Fernández, 2018) pues el contacto con el medio y sus elementos favorece la adquisición de conocimientos de forma significativa (García de la Vega, 2012; Tejeda, 2009) y "contribuye a la transversalidad de la enseñanza" (Fernández, 2018: 883). Igualmente, este instrumento resulta efectivo para que los estudiantes puedan comprender las interrelaciones existentes entre los elementos que integran el espacio geográfico y cómo ello puede incidir en la evolución del paisaje (García de la Vega, 2012; Hernández et al., 2015; Hernández y Rivero, 2015; Thomashow, 1995; TorresPorras, et al., 2017). El contacto con el medio natural a través de la excursión hace que los estudiantes desarrollen conciencia ambiental (Granados, 2011: Liceras, 2003; 2013) y sentimiento de identidad con el territorio vivido y percibido (Hernández, 2009; Gómez-Mendoza, 2013; Fernández, 2015). Para ello, los geoparques ponen a disposición de los docentes la infraestructura necesaria para que en contacto con la naturaleza puedan trabajarse contenidos de las diferentes materias que integran la Educación Primaria (Azman et al., 2010; Fernández, 2018). Los geoparques son "áreas geográficas únicas y unificadas donde los sitios y paisajes de importancia geológica internacional se gestionan como un concepto holístico de protección, educación y desarrollo sostenible" (UNESCO, 2019). La UNESCO (2017) los define como aquellos espacios que poseen unas características, ya sean geológicas o geomorfológicas, que los singularizan e individualizan y que, además, tienen unos límites claramente 


\section{Rubén Fernández Álvarez}

definidos y suficiente superficie para promover estrategias de desarrollo económico que estarán articuladas por dos ejes de actuación: educativo y turístico. En su enfoque combina el desarrollo socioeconómico con la conservación de los recursos territoriales e incluye a la ciudadanía en los procesos de gestión y de toma de decisiones (López, 2016; McKeever y Zouros, 2005). Los geoparques se han convertido en una alternativa para los espacios rurales, un nuevo instrumento definido para fomentar la cohesión territorial y el desarrollo socioeconómico de las áreas más desfavorecidas (Zouros, 2004). Para conseguir los objetivos propuestos por este elemento se han determinado dos líneas de acción preferentes: el geoturismo y la geoeducación (Azman et al., 2010). El geoturismo es un concepto relativamente novedoso (Hose, 2012; Newsome y Dowling, 2010; Ólafsdóttir y Tverijonaite, 2018) con el que se trata de identificar a aquellas acciones turísticas cuyo centro de acción se circunscribe a la geología o a la geomorfología. Esta tipología turística de carácter especializado en la que la ampliación de conocimientos adquiere un destacado papel, hace que la vertiente educativa, ya sea de tipo formal o informal, alcance una posición determinante (Dowling, 2013; Farsani, et al., 2017; Justice, 2018; Lewis y Hampton, 2015; Mikhailenko y Ruban, 2019; Newsome y Dowling, 2010; Pforr y Megerle, 2006). Por su parte, la geoeducación comienza a ganar relevancia en los contextos de los geoparques. Esta figura dispone de unas características geológicas y ambientales que la singularizan y la convierten en centro de atracción de turistas "educativos". Con ello se produce una transferencia de conocimientos que revierte en el incremento de la concienciación ambiental (Farsani, et al., 2017) y en la formación de la ciudadanía que habita en el radio de acción de los geoparques (Justice, 2018). Cada vez son más las iniciativas de tipo educativo que se ofertan desde los geoparques para poner en valor los recursos paisajísticos, geológicos y geomorfológicos que poseen estos territorios (Farsani et al., 2014). Según el estudio realizado por Farsani et al. (2014) los geoparques adaptan sus propuestas educativas para que sean accesibles a todo tipo de usuarios, pasando de la información geológica especializada a la información geológica común. Con los mismos postulados Bobrowsky et al. (2017) afirman que los geocientíficos son fundamentales para que la sociedad pueda llegar a conocer las características de los recursos geológicos existentes en su territorio. En este sentido, uniendo las ideas de ambos trabajos (Bobrowsky et al., 2017; Farsani et al., 2014), la combinación geoparques y expertos en la materia pueden dar como resultado un profundo conocimiento de la geología y del territorio por parte de la ciudadanía si se crean los canales de transferencia adecuados.

Los geoparques se conciben, además de como elementos para diversificar económicamente los lugares sobre los que se ubican, como espacios en los que crear conocimientos y generar una concienciación ambiental en la ciudadanía (Dowling, 2013; Eder y Patzak, 2004; Farsani et al 2013; González-Tejeda et al., 2017; Ngwira, 
2015; Wojtowicz y Wojtowicz, 2016; Zouros y Martini, 2003). Por ello, entre sus líneas de acción se han de destacar las que están centradas tanto en las colaboraciones de investigación con universidades y centros de investigación como las que lo están en la formación a través del trabajo conjunto con los centros educativos del contexto territorial más cercano. Para dar cabida a este último aspecto, los geoparques han de desarrollar un proyecto educativo sólido en el que se plasmen las iniciativas didácticas que van a poner en marcha, además de contar con las infraestructuras y el personal adecuado para que estos puedan ejercer de núcleo de transmisión de conocimientos (Bitschene y Schüller, 2011). Aunque a través de estos medios es posible el aprendizaje autónomo, los geoparques presentan líneas de actuación en el ámbito educativo formal, desarrollándose una estrecha colaboración entre estos y los centros docentes. Así, se está favoreciendo el acceso a los conocimientos medioambientales y geográficos desde la base educativa y creando una sociedad con cierto nivel de concienciación, no solo geológico, sino ambiental y paisajístico (Catana y Rocha, 2009; Farsani et al., 2017; Mieczkowski, 1995; Ólafsdóttir y Tverijonaite, 2018; Rodrigues y Neto, 2009). Además, la población que habita en los geoparques necesita conocer en profundidad el territorio y sus recursos para ofrecer al visitante un mejor servicio, para poder participar en las iniciativas de gestión y de protección y para entender las repercusiones socioeconómicas que esta figura puede tener sobre el espacio. Se puede considerar que los geoparques son espacios que reciben, por parte de la UNESCO, esta calificación en virtud de sus características geológicas y geomorfológicas. Sin embargo, entre las exigencias de la Organización de Naciones Unidas figuran aquellas que tienen que ver con la educación y el desarrollo económico. Tal y como se indicaba con anterioridad todos los geoparques tienen que contar con un detallado proyecto educativo orientado, tanto a escolares de los colegios de su área de acción como al público en general (UNESCO, 2017).

Con el desarrollo de la presente investigación se persiguen una serie de objetivos, siendo el de carácter general el centrado en el estudio, valoración y caracterización de los geoparques españoles reconocidos por la UNESCO como elementos didácticos para la etapa de Educación Primaria, para así, poder reconocer a esta figura como un notable elemento de valor educativo para poner en marcha y desarrollar en ellos procesos de enseñanza-aprendizaje de los paisajes de las áreas de montaña. De forma específica, para apoyar al general, se plantean una serie de objetivos complementarios, todos ellos centrados en la incorporación del paisaje y del paisaje de montaña en los contenidos que se detallan en los marcos normativos nacionales y autonómicos de educación, más concretamente, de Educación Primaria (EP). De este modo, se pretende analizar los contenidos en materia de paisaje para identificar cuáles de ellos pudieran trabajarse desde los geoparques. 


\section{METODOLOGÍA Y MATERIALES}

El desarrollo del presente trabajo se articula a partir de una metodología cualitativa e interpretativa basada en la técnica de análisis de documentos (Hoepfl, 1997; Massot et al., 2004; Pérez et al., 2012). Se trata de un método de inducción de categorías temáticas (Pérez et al, 2012) que se lleva a cabo desde las referencias explícitas localizadas en los documentos analizados. Este proceso se ha realizado mediante el uso del software MAXQDA 18.2, con él se ha procedido a la búsqueda y el análisis en el texto de una serie de categorías temáticas que han permitido, por un lado, caracterizar las actividades educativas llevadas a cabo en los geoparques y, por el otro, identificar la presencia del paisaje en los documentos curriculares de las autonomías que cuentan con un geoparque en su territorio. Así, para ello se han definido dos sistemas de categorías: uno basado en la Carta Europea de los Geoparques y otro apoyado en los postulados emanados del Convenio Europeo del Paisaje (CEP).

Tras una primera fase de análisis de los documentos de los geoparques se han identificado cinco grandes categorías, cuatro de ellas de carácter temático y una quita para identificar los métodos de enseñanza: Elementos territoriales; Geología; Elementos antrópicos; Acciones para la concienciación; Educación (materiales y métodos de las actividades). Todas ellas están en consonancia con los postulados educativos para los que fueron definidos los geoparques y siguen las ideas principales de la Carta Europea de los Geoparques: geoconocimiento; concienciación ambiental; conocimiento cultural y territorial. Cada una de estas categorías se encuentra integrada por palabras clave que posibilitan identificar con precisión las características de la documentación y de las actividades y, a su vez. posibilitarán reconocer las temáticas secundarias que son incorporadas de forma transversal en las actividades.

La segunda fase de análisis se ha centrado en los documentos curriculares autonómicos. Para esta se ha definido un sistema de categorías articulado por los postulados emanados del CEP: Concepto de paisaje; Diversidad de paisajes; Paisajes naturales; Paisajes antrópicos; Gestión y conservación del paisaje; Evolución y transformación del paisaje. Con ello se pretende definir un hilo conductor que permita relacionar los contenidos que aparecen en la normativa con los que pueden ser trabajados desde las actividades de los geoparques.

Los materiales utilizados para el análisis de las actividades ofertadas por los geoparques a través de su proyecto educativo se centran, principalmente, en las páginas web de los geoparques y en todo el material disponible para su descarga o consulta en red. Con ello se ha podido acceder a la oferta educativa, tanto formal como no formal, que los geoparques ponen a disposición de los usuarios. Igualmente, en los que está disponible, se ha accedido al proyecto educativo y a la memoria de actividades. Todo ello nos permite caracterizar la tipología de las actividades, así como la etapa a la que van dirigidas y si existe una incorporación explícita en ellas de la enseñanza-aprendizaje 
del paisaje. En el ámbito legislativo los documentos analizados son los currículos de las comunidades autónomas en las que hay un geoparque (ver tabla 1), más concretamente los apartados de estos centrados en los contenidos de la materia de Ciencias Sociales. Con estos dos bloques de materiales se puede obtener la presencia del paisaje de forma explícita entre los contenidos curriculares y la orientación de las actividades propuestas por los geoparques y cómo el paisaje aparece en ellas.

\begin{tabular}{|c|c|}
\hline $\begin{array}{l}\text { Comunidad autónoma y } \\
\text { geoparque }\end{array}$ & Documento normativo \\
\hline $\begin{array}{l}\text { Andalucía (Cabo de Gata- } \\
\text { Níjar; Sierras Subbéticas; } \\
\text { Sierra Norte de Sevilla) }\end{array}$ & $\begin{array}{c}\text { DECRETO 97/2015, de } 3 \text { de marzo, por el que se establece } \\
\text { la ordenación y el currículo de la Educación Primaria en la } \\
\text { Comunidad Autónoma de Andalucía. }\end{array}$ \\
\hline Aragón (Sobrarbe-Prineos) & $\begin{array}{l}\text { ORDEN de } 16 \text { de junio de 2014, de la Consejera de Educación, } \\
\text { Universidad, Cultura y Deporte, por la que se aprueba el } \\
\text { currículo de la Educación Primaria y se autoriza su aplicación en } \\
\text { los centros docentes de la Comunidad Autónoma de Aragón. }\end{array}$ \\
\hline $\begin{array}{l}\text { Castilla-La Mancha } \\
\text { (Molina y Alto Tajo) }\end{array}$ & $\begin{array}{c}\text { DECRETO 54/2014, de 10/07/2014, por el que se establece el } \\
\text { currículo de la Educación Primaria en la Comunidad Autónoma } \\
\text { de Castilla-La Mancha }\end{array}$ \\
\hline \multirow[b]{2}{*}{ Castilla y León (Las Loras) } & $\begin{array}{l}\text { ORDEN EDU/519/2014, de } 17 \text { de junio, por la que se establece } \\
\text { el currículo y se regula la implantación, evaluación y desarrollo } \\
\text { de la educación primaria en la Comunidad de Castilla y León }\end{array}$ \\
\hline & $\begin{array}{l}\text { ORDEN EDU/278/2016, de } 8 \text { de abril, por la que se modifica la } \\
\text { Orden EDU/519/2014, de } 17 \text { de junio, por la que se establece el } \\
\text { currículo y se regula la implantación, evaluación y desarrollo de } \\
\text { la educación primaria en la Comunidad de Castilla y León }\end{array}$ \\
\hline $\begin{array}{l}\text { Cataluña (Cataluña Ce } \\
\text { Conca de Tremp-Mor }\end{array}$ & $\begin{array}{c}\text { DECRETO 119/2015, de } 23 \text { de junio, de ordenación de las } \\
\text { enseñanzas de la educación primaria. }\end{array}$ \\
\hline $\begin{array}{l}\text { Extremadura (las } \\
\text { Villuercas) }\end{array}$ & $\begin{array}{l}\text { DECRETO 103/2014, de } 10 \text { de junio, por el que se establece el } \\
\text { currículo de Educación Primaria para la Comunidad Autónoma } \\
\text { de Extremadura. }\end{array}$ \\
\hline $\begin{array}{l}\text { Islas Canarias (El Hierro; } \\
\text { Lanzarote y Archipiélago } \\
\text { Chinijo) }\end{array}$ & $\begin{array}{c}\text { DECRETO 89/2014, de } 1 \text { de agosto, por el que se establece } \\
\text { la ordenación y el currículo de la Educación Primaria en la } \\
\text { Comunidad Autónoma de Canarias. }\end{array}$ \\
\hline País Vasco (Costa Vasca) & $\begin{array}{c}\text { DECRETO 236/2015, de } 22 \text { de diciembre, por el que se establece } \\
\text { el currículo de Educación Básica y se implanta en la Comunidad } \\
\text { Autónoma del País Vasco. }\end{array}$ \\
\hline
\end{tabular}

TABLA $\mathrm{N}^{\mathrm{O}} 1$. Normativa curricular autonómica

Fuente: elaboración propia 


\section{EL PAISAJE EN EDUCACIÓN PRIMARIA}

El 20 de octubre del año 2000, en la ciudad de Florencia, se firma en el seno del Consejo de Europa el Convenio Europeo del Paisaje (CEP), aunque su puesta en funcionamiento se retrasará hasta el 01 de marzo de 2004. Nos encontramos ante un documento normativo que otorga al paisaje un atributo jurídico, es de carácter transnacional y se diseña con el objeto de fomentar medidas para la protección, gestión y ordenación del paisaje (Zoido, 2004; Ortega, 2007; Nogué, et al., 2010; Fernández, 2015).

Aunque España formó parte del grupo de países firmantes del CEP en el año 2000, no será hasta noviembre de 2007 cuando se produzca su ratificación y su posterior entrada en vigor el 01 de marzo de 2008. En este sentido, el Estado ha de adaptarse a los postulados emanados del CEP: identificación, gestión, ordenación y educación en temática paisajística (Consejo de Europa, 2000). Es de reseñar que en el presente Convenio el apartado centrado en la educación en materia de paisaje, se incluye concretamente en su artículo sexto, apartado B, que aparece redactado en los siguientes términos:

"Formación y educación. Cada parte se compromete a promover: a) la formación de especialistas en la valoración de los paisajes e intervención en los mismos; b) programas pluridisciplinares de formación en política, protección, gestión y ordenación de paisajes con destino a los profesionales de los sectores privado y público y a las asociaciones interesadas; c) cursos escolares y universitarios que, en las disciplinas correspondientes, aborden los valores relacionados con los paisajes y las cuestiones relativas a su protección, gestión y ordenación" (Consejo de Europa, 2000, p. 8). En relación con las características analizadas en la presente investigación, enseñanza del paisaje será en el artículo sexto, en su apartado B,c, en el que debemos centrar nuestra atención, pues está referido a la enseñanza escolar. Este pone de manifiesto que se ha de formar a la ciudadanía desde la base educativa trabajando, tanto los valores paisajísticos como aquello que tienen que ver con la gestión y la protección del paisaje (Busquets, 2010; Calcagno, 2016). Es decir, los "escolares" han de recibir en su proceso formativo contenidos referidos al paisaje. En el momento en el que España se adhiere a este Convenio adquiere un compromiso que le insta a trabajar el paisaje en las aulas. Actividad que puede desarrollarse desde la educación ambiental y desde el trabajo de campo en contacto con el medio (Casas y Erneta, 2015)

Aunque es este artículo sexto el que hace mención explícita a la formación y educación, también en la propia definición de paisaje aportada por el CEP se puede extraer una referencia al proceso formativo. Puntualizamos que existe una referencia implícita a la educación en esta definición (Calcagno, 2016), porque se considera que en el momento en el que una persona tenga que describir su percepción a cerca de lo que observa necesita una mínima formación para poder desarrollar adecuadamente este proceso. 
No se debe pasar por alto que el CEP ofrece pautas, tales como la inclusión del paisaje en la enseñanza, pero a su vez, otorga libertad a los países firmantes para que adapten la enseñanza del mismo y desarrollen los mecanismo más oportunos en función de sus singularidades sociales, paisajísticas y educativas (Zoido, 2006b). Estamos ante un documento normativo que sienta las bases de actuación en materia paisajística y que lo hace de forma integral. Proporciona una definición de "paisaje", incide en la importancia de obtener el carácter del paisaje (identificar aquellos elementos que dan carácter a un paisaje y que, a la postre, lo individualizan y singularizan aportándole una identidad propia) para gestionarlo y ordenarlo en función de sus necesidades e indica que se ha de formar a la población en paisaje (formación articulada por una doble vertiente: base educativa y profesionales del ámbito público y privado).

\subsection{El paisaje en la normativa nacional de educación: Real Decreto 126/2014, de 28 de febrero, por el que se establece el currículo básico de la Educación Primaria.}

En el ámbito nacional será el Real Decreto 126/2014, de 28 de febrero, por el que se establece el currículo básico de la Educación Primaria, el analizado con el objeto de estudiar la incorporación del paisaje a los contenidos mínimos que han de aprender los alumnos de esta etapa educativa.

En este sentido, la primera interpretación en cuanto a temáticas referidas al paisaje podría encontrarse en el artículo 7 del presente documento, "objetivos de la Educación Primaria”, pues en su apartado "h" define el siguiente objetivo: "Conocer los aspectos fundamentales de las Ciencias de la Naturaleza, las Ciencias Sociales, la Geografía, la Historia y la Cultura" (BOE, 2014, p. 19.354). De este modo, al identificar como objetivo el conocimiento de los aspectos fundamentales de la Geografía, se está haciendo alusión al paisaje, pues este es el exponente visual del espacio geográfico (Miralbés e Higueras, 1993). Volviendo a la definición que aporta el CEP sobre este elemento "-cualquier parte del territorio tal como la percibe la población-" se podría fijar una primera relación entre el Convenio y la Normativa en materia de educación. El primero hace referencia a la percepción visual que la población tiene del territorio y el segundo indica que se han de conocer los aspectos fundamentales de la Geografía, siendo uno de estos aspectos el "paisaje". A la postre, en ambos casos se está aludiendo al elemento paisaje.

De forma específica el término que nos ocupa aparece por primera vez en el Anexo I (apartado encargado de la descripción de las asignaturas troncales que integran la enseñanza de la EP), concretamente en la asignatura troncal "Ciencias Sociales", donde se puede leer lo siguiente: "Se identificarán los elementos del paisaje (relieve, 
clima, hidrografía) y se describirán y caracterizarán los principales medios naturales y su localización. Por último, se analizará la influencia humana en el medio y sus consecuencias ambientales" (BOE, 2014, p. 19.372). De este modo, se está proponiendo el estudio de los elementos que integran el paisaje, así como, la presencia humana en el medio, influencia que se verá reflejada en el carácter evolutivo del paisaje.

Centrando la atención en los contenidos que define el presente Real Decreto, la incorporación del paisaje a los mismos no se da hasta el bloque 2 "el mundo en que vivimos". Así, este bloque segundo fija los dos siguientes contenidos: a) La diversidad geográfica de los paisajes de España: relieve e hidrografía. b) La diversidad geográfica de los paisajes de Europa: relieve, climas, e hidrografía (BOE, 2014, p. 19.375). En los dos casos se centra en aspectos físicos del territorio, siendo elementos tales como el relieve, el clima y la hidrología los que aparecen destacados. De este modo, propone el trabajo, tanto de las particularidades paisajísticas de España como de Europa, integrando la caracterización del paisaje en un contexto territorial mayor. Es decir, no solo se trabaja la diversidad española sino que se trabaja también la europea, aspecto este que sirve para enmarcar la diversidad nacional en un marco de referencia territorial más amplio. A este respecto, estos dos contenidos enlazan con los postulados del Convenio Europeo del Paisaje que propone que el análisis del paisaje se haga de forma integral y en su totalidad, desde lo general a lo particular (Busquets y Cortina, 2016).

Para estos contenidos se definen los siguientes criterios de evaluación y estándares de aprendizaje evaluables: "Explicar que es un paisaje e identificar los principales elementos que lo componen". "Define paisaje, identifica sus elementos y explica las características de los principales paisajes de España y Europa, valorando su diversidad' (BOE, 2014, p. 19.375). Por ello, se entiende que al finalizar la etapa de primaria los estudiantes han adquirido las capacidades y destrezas suficientes para definir el término paisaje y para identificar y explicar los elementos que lo componen, especialmente aquellos que tienen que ver con los aspectos físicos del territorio, tanto en los referidos a España como en los de Europa.

\subsection{El paisaje en la normativa de educación de las Comunidades Autónomas}

En el ámbito autonómico, cada una de las Comunidades deberá desarrollar el documento por el que se implanta el currículo en base a lo dictado por el Real Decreto 126/2014, de 28 de febrero, por el que se establece el currículo básico de la Educación Primaria. En este sentido, la administración regional podrá incluir aquellos contenidos que considere necesarios en función de sus particularidades geográficas, sociales, históricas y culturales. Por ello, en este apartado se pretende 
analizar los instrumentos legislativos utilizados por las administraciones autonómicas que disponen de un geoparque en su territorio ${ }^{2}$, con el objeto de estudiar si, de algún modo, se ve modificado (ampliado) el proceso de enseñanza-aprendizaje del paisaje en lo que a los contenidos se refiere.

Entre la normativa recogida de las diferentes autonomías, siete de las ocho cuentan con referencias expresas al paisaje en su marco legal diseñado para la implantación de la EP. En este sentido, Aragón, Castilla -La Mancha, Castilla y León, Cataluña, Extremadura, Islas Canarias y País Vasco, además de los contenidos que vienen determinados por la normativa nacional, añaden contenidos relativos a esta materia. Es decir, proponen un mayor número de conocimientos relacionados con el paisaje.

De este modo, las siete Comunidades anteriormente citadas distribuyen entre los seis cursos de la etapa los siguientes contenidos sobre el paisaje ${ }^{3}$ :

a) Los paisajes: elementos naturales y humanos del entorno (diversidad de paisaje natural y diversidad de paisaje humanizado).

b) Los paisajes de interior y de costa (llanura, montaña y costa).

c) Diversidad de paisajes e influencia humana.

d) Diversidad de paisajes de cada Autonomía.

e) Diversidad paisajística de España.

f) Diversidad paisajística de Europa.

g) Respeto, defensa y mejora del paisaje (concienciación y conservación) en Aragón, Castilla -La Mancha e Islas Canarias.

Siguiendo las líneas que propone el Convenio Europeo del Paisaje, en todas las Comunidades Autónomas adquieren una importancia notable los aspectos relacionados con la humanización del paisaje, las transformaciones resultantes y la evolución del medio. Se han de trabajar una serie de contenidos centrados en ello con el objeto de que los estudiantes de primaria, al final de su etapa, hayan adquirido la capacidad de discernir en la configuración del paisaje entre la presencia de elementos naturales y antrópicos. Igualmente, deberán estar capacitados para analizar y valora la intervención humana en el medio y su respuesta en la evolución y transformación del paisaje.

2 Cuentan con al menos un geoparque en su territorio las siguientes Comunidades Autónomas: Andalucía, Aragón, Castilla -La Mancha, Castilla y León, Cataluña, Extremadura, Galicia, Islas Canarias y País Vasco.

3 No se referencian tal y como aparecen citados en los diferentes instrumentos curriculares, para ello se ha definido una denominación que agrupe-resuma por aproximación los contenidos que proponen y así evitar enumerar todos ellos y caer en repeticiones. 
A partir de los contenidos centrados en la humanización del paisaje, surgen en tres Comunidades Autónomas (Aragón, Castilla -La Mancha e Islas Canarias) los que tienen que ver con el respeto, defensa y mejora del paisaje. A este respecto, también siguiendo las premisas del CEP, se incorporan los matices relacionados con la adquisición de valores centrados directamente en el respeto y la conservación del paisaje. En cambio, en el resto de regiones, aunque no existan estos contenidos de forma específica, se entiende que también están presentes de forma indirecta. En este sentido, se adquieren los valores de respeto y conservación a partir del trabajo con los contenidos centrados en la conservación del medio ambiente.

En lo que al paisaje de montaña se refiere, en tres de los instrumentos autonómicos (Aragón, Castilla -La mancha y Extremadura) se hace referencia de forma explícita a ello. De este modo, entre los contenidos figuran aquellos que se centran en los paisajes de las áreas de montaña, así como en los elementos que los integran y su evolución.

En el resto de las autonomías, más bien, las referencias están orientadas al aprendizaje de los elementos naturales que configuran el paisaje. En este caso, en el momento en el que se trabaja el relieve y su aportación a la construcción del paisaje, uno de los apartados ha de ser el relativo a los relieves de montaña, pues se tienen en consideración la diversidad de paisajes de interior, conjunto del que forma parte.

En el caso específico de la Comunidad Autónoma de Andalucía, en el documento elaborado para fijar el currículo de Educación Primaria no se ha detectado mención alguna al paisaje, en este sentido entendemos que no cuentan con ningún contenido más a este respecto que aquellos que vienen determinados por el Gobierno Central en su marco normativo de educación.

\section{DEFINICIÓN Y CARACTERIZACIÓN DE LA FIGURA “GEOPARQUE”}

A partir del desarrollo, en el año 2000, de un proyecto de cooperación europeo LEADER, se configura la red European Geoparks Network (EGN), integrada, en un primer momento, por un total de cuatro territorios singularizados por sus características geológicas y geomorfológicas, además de por su calidad ambiental y paisajística y sus virtudes culturales: Parque Cultural del Maestrazgo (España), Reserva Geológica de la Alta Provenza (Francia), Geoparque Vulkaneifel (Alemania) y Bosque Petrificado de Lesbos (Grecia) (EGN, 2017; Farsani et al., 2011; López 2016). La EGN se consolida como un eje de trabajo común orientado al fomento de la educación en materia ambiental y territorial, al desarrollo endógeno y a la diversificación económica y al impulso e intercambio científico. Nos encontramos ante un elemento que aúna educación y ciencia junto con desarrollo económico, es decir, a partir de una serie de recursos territoriales trata de fomentar el desarrollo endógeno canalizándolo a través 
de la educación y del intercambio científico. En este sentido se va a promocionar el turismo de tipo educativo (Farsani, et al., 2011; Fernández, 2016; López, 2016; Ólafsdóttir y Dowling, 2014; Zouros, 2010) con el objeto de concienciar a la población desde la base educativa en materia ambiental, paisajística y cultural.

Tras la fundación de EGN, en el año 2001 se acuerda que la figura geoparque pase a formar parte de la División de las Ciencias de la Tierra de la UNESCO dando lugar, en el año 2004, a la puesta en marcha de Global Geoparks Network (GGN) (EGN, 2017) que desemboca, ya en el año 2015, en la figura UNESCO Global Geoparks y se incorpora al Programa Internacional Ciencias de la Tierra y Geoparques.

En el marco de los ejes turismo y educación la UNESCO propone que las áreas de trabajo que han de dinamizar todos los territorios que aspiren a formar parte de esta red, sin excepción alguna, son las que se enumeran a continuación: recursos naturales, peligros geológicos, cambio climático, educación, ciencia, cultura, mujer, desarrollo sostenible, cultura tradicional, geoconservación (EGN, 2017; UNESCO, 2017). Se trata de diez áreas en las que los procesos educativos se consideran la base fundamental, trabajando todas ellas desde la educación y la enseñanza. De este modo, es posible llagar a la concienciación en materia de protección, conservación y gestión ambiental (Azma et al., 2010). Además, se ha de resaltar que las propuestas educativas que cada geoparque realice deberán ser de carácter transversal e incluir, en lo posible, la mayor parte de las áreas anteriormente identificadas (UNESCO, 2017).

Aunque en un principio la percepción que se obtiene de esta figura es que se define para el estudio de la geología, una vez analizadas sus áreas y temáticas de actuación, se ha de entender como un recurso que va más allá y aspira a convertirse en un entorno de enseñanza integral, tanto del espacio geográfico como de la educación en valores.

Igualmente, la figura geoparque requiere la elaboración y el desarrollo de un "Proyecto Educativo" en el que se plasmen, de forma detallada, aquellas medidas o iniciativas que se van a llevar a cabo como instrumentos de enseñanza y formación.

La red Global Geopark Network está formada por un total de 147 geoparques (mayo de 2019) repartidos entre África (2), América (9), Asía (58) y Europa (78) (ver figura $\mathrm{n}^{\mathrm{o}} 1$ ). Del total, cuatro de ellos son de carácter transnacional compartidos entre Austria y Eslovenia (Karavanke UNESCO Global Geopark), Alemania y Polonia (Muacau Arch UNESCO Global Geopark), Hungría y Eslovaquia (Novohrad-Nógrád UNESCO Global Geopark) y entre Irlanada y el Reino Unido (Marble Arch Caves UNESCO Global Geopark). 


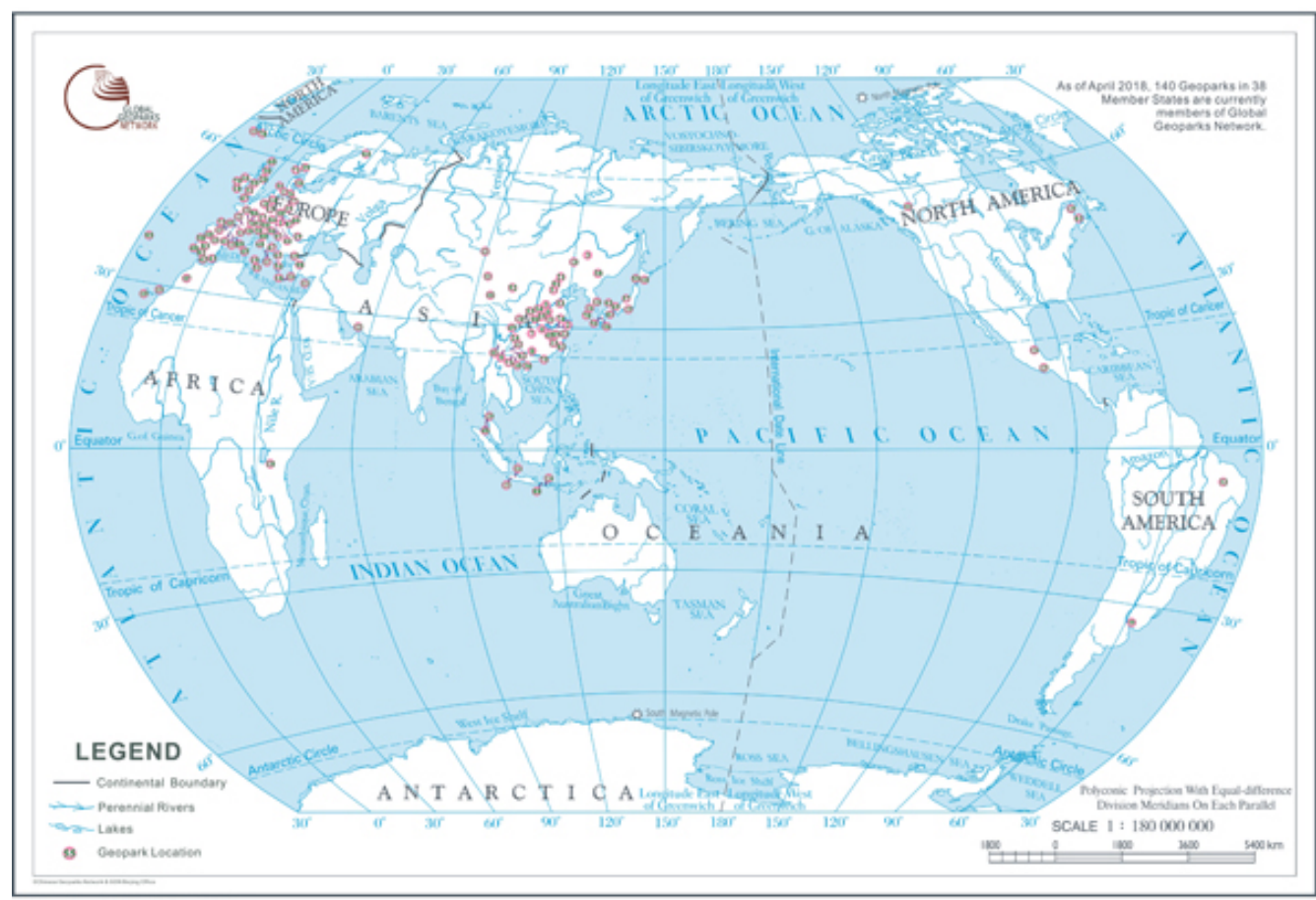

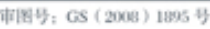

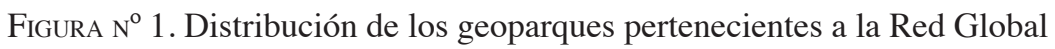
Fuente: Global Geopark Network

\section{LOS GEOPARQUES ESPAÑOLES}

De entre los geoparques mundiales de la UNESCO (ver figura 1), un total de trece están ubicados en España (ver tabla $n^{\circ}$ 2), siendo el segundo país a escala global con mayor número por detrás de China (39 geoparques). La superficie ocupada del territorio nacional por esta figura asciende a $19.923 \mathrm{~km}^{2}$ aproximadamente el $3,5 \%$ del total.A priori, aunque pueda parecer un porcentaje relativamente pequeño para el conjunto nacional, se ha de resaltar que todo ese espacio está destinado al fomento de actividades, entre otras, educativas. Igualmente, con ello se pretende crear redes nacionales e internacionales de intercambio de conocimientos y experiencias y definir una fórmula común con el objeto de unificar planteamientos en cuanto a la orientación de las propuestas educativas a desarrollar, siempre teniendo en cuenta las singularidades de cada territorio y de su población. 


\begin{tabular}{|c|c|c|c|c|}
\hline Denominación & Provincia & C. Autónoma & $\begin{array}{l}\text { Superficie } \\
\mathbf{k m}^{2}\end{array}$ & $\begin{array}{l}\text { Año de } \\
\text { incorporación } \\
\text { a la red global }\end{array}$ \\
\hline Geoparque Cabo de Gata -Níjar & Almería & Andalucía & 500 & 2006 \\
\hline $\begin{array}{l}\text { Geoparque de las Sierras } \\
\text { Subbéticas }\end{array}$ & Córdoba & Andalucía & 320 & 2006 \\
\hline Geoparque Sobrarbe-Pirineos & Huesca & Aragón & 2.202 & 2006 \\
\hline $\begin{array}{l}\text { Geoparque Costa Vasca / } \\
\text { Geoparkea Euskal Kostaldea }\end{array}$ & Guipúzcoa & País Vasco & 89 & 2010 \\
\hline $\begin{array}{l}\text { Geoparque Sierra Norte de } \\
\text { Sevilla }\end{array}$ & Sevilla & Andalucía & $1.774,84$ & 2011 \\
\hline $\begin{array}{l}\text { Geoparque Villuercas-Ibores- } \\
\text { Jara }\end{array}$ & Cáceres & Extremadura & $2.544,40$ & 2011 \\
\hline $\begin{array}{l}\text { Geoparque Cataluña Central / } \\
\text { Geoparc de la Catalunya Central }\end{array}$ & Barcelona & Cataluña & 1.300 & 2012 \\
\hline $\begin{array}{l}\text { Geoparque de Molina y Alto } \\
\text { Tajo }\end{array}$ & Guadalajara & $\begin{array}{l}\text { Castilla-La } \\
\text { Mancha }\end{array}$ & 4.520 & 2014 \\
\hline Geoparque El Hierro & Tenerife & Islas Canarias & 595 & 2014 \\
\hline $\begin{array}{l}\text { Geoparque Lanzarote y } \\
\text { Archipiélago Chinijo }\end{array}$ & Gran Canaria & Islas Canarias & 2.500 & 2015 \\
\hline Geoparque Las Loras & $\begin{array}{l}\text { Burgos/ } \\
\text { Palencia }\end{array}$ & $\begin{array}{l}\text { Castilla y } \\
\text { León }\end{array}$ & 951 & 2017 \\
\hline $\begin{array}{l}\text { Geoparque Conca de Tremp- } \\
\text { Montsec }\end{array}$ & Lleida & Cataluña & 2.050 & 2018 \\
\hline Geoparque Montaña do Courel & Lugo & Galicia & 578 & 2019 \\
\hline
\end{tabular}

Superficie total geoparques

$19.923,85$

TABLA N $^{\circ}$ 2. Geoparques españoles. Fuente: UNESCO Geoparks

Desde la puesta en marcha de la red Geoparques en el año 2000 España siempre ha contado en su territorio con alguna figura de estas características. El antiguo geoparque del Maestrazgo, en la actualidad Parque Cultural del Maestrazgo, fue uno de los cuatro que pusieron en marcha esta red. Este no ha podido mantener su catalogación debido a la pérdida de uno de los requisitos básicos que define la UNESCO (apoyo y confianza de la Administración nacional). Por ello, en la actualidad son trece los geoparques que se encuentran en funcionamiento en España bajo el amparo de la UNESCO e integrados en la red global.

Se distribuyen por siete comunidades autónomas y once provincias (ver figura $\mathrm{n}^{\mathrm{o}} 2$ ), cubriendo la totalidad del espacio administrativo de 238 municipios. 


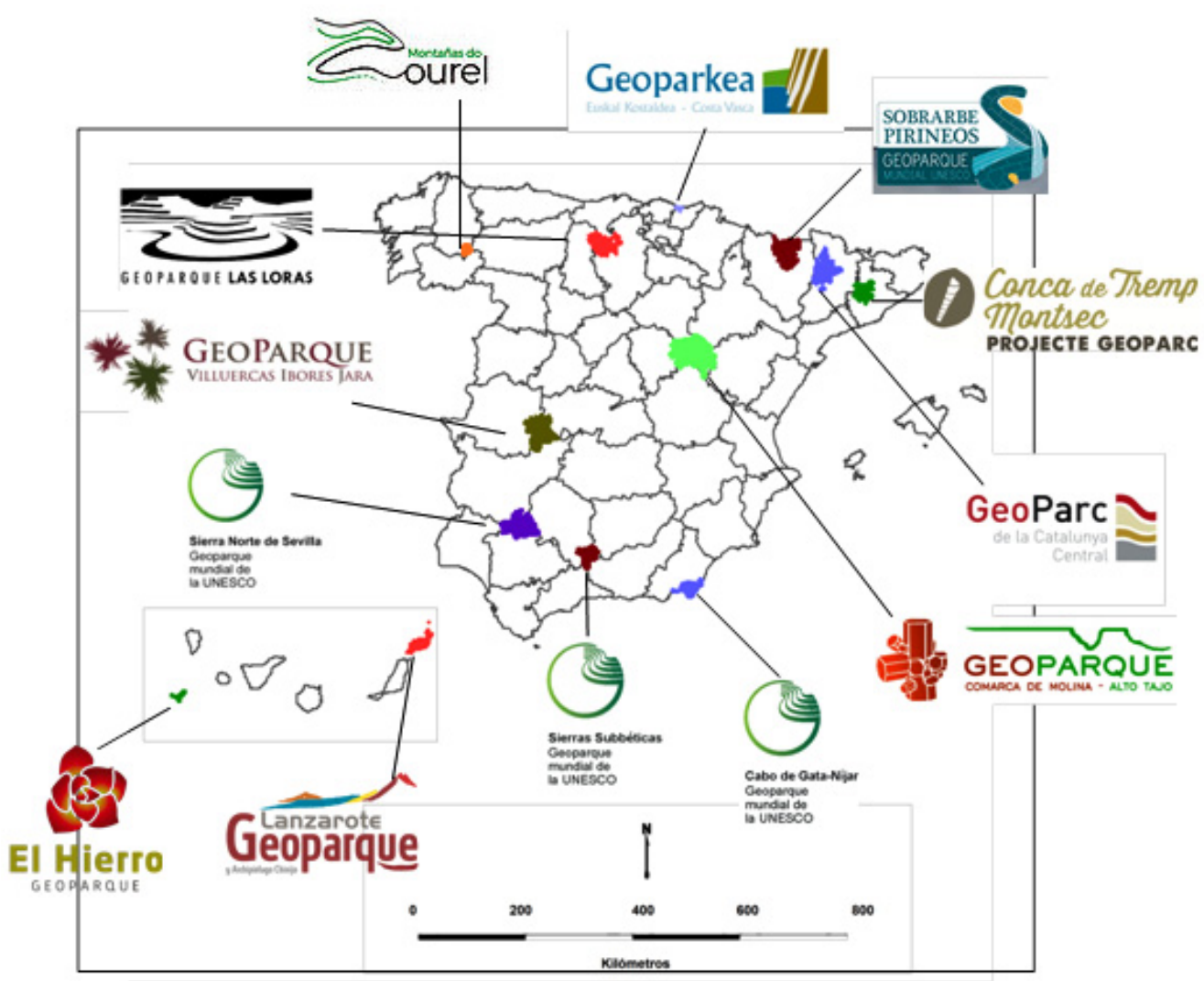

FIGURA N ${ }^{0}$ 2. Localización de los Geoparques Globales de la UNESCO en España Fuente: elaborado a partir de UNESCO Geoparks y Webs de los geoparques.

Con ello, estos ponen a disposición de los usuarios en torno a 545 geositios de características geológicas, geomorfológicas, paisajísticas, culturales ambientales, etc. muy diversas que son unidos por 172 rutas de tipo terrestre y marino, todas ellas accesibles a todos los públicos, aunque su componente educativo hace que sean los centros formativos los que hagan un mayor uso de estos espacios.

Bajo su área de influencia ${ }^{4}$ se contabilizan 753.581 habitantes (ver tabla $n^{\circ} 3$ ), con una densidad media de población 38,9 hab $/ \mathrm{km}^{2}$. Disponen de una densidad media para el

${ }^{4}$ En este caso se tiene en consideración, a la hora de determinar el área de influencia, solamente a los municipios que se encuentran bajo el espacio ocupado por el geoparque. Se seleccionan estos municipios porque son los que disponen de atención preferente para el desarrollo de las actividades ofertadas por los geoparques. A pesar de ello, cualquier centro educativo, independientemente de su procedencia, puede hacer uso de ellos. 
conjunto relativamente baja (media nacional en torno a $92 \mathrm{hab} / \mathrm{km}^{2}$ ) y, además, existe un marcado desequilibrio entre unos geoparques y otros. Así, mientras en el Geoparque Cabo de Gata-Níjar (integrado por los municipios de Almería, Carboneras y Níjar) se registra una densidad de población cercana a los 450 hab $/ \mathrm{km}^{2}$, en el Geoparque Sobrarbe-Pirineos (19 municipios del norte de la provincia de Huesca) la densidad es de 3,3 hab/ $\mathrm{km}^{2}$. Este contraste viene determinado por el ámbito urbano del primero frente al rural del segundo.

\begin{tabular}{|c|c|c|c|c|c|c|c|c|}
\hline Geoparques & $\begin{array}{l}\text { Número de } \\
\text { municipios }\end{array}$ & $\begin{array}{c}\text { Superficie } \\
\mathrm{km}^{2}\end{array}$ & Población & $\begin{array}{c}\text { Población } 6 \text { - } \\
12 \text { años }\end{array}$ & $\begin{array}{c}\text { Número de } \\
\text { colegios (EP) }\end{array}$ & $\begin{array}{c}\text { Densidad de } \\
\text { población } \\
\left(\mathrm{hab} / \mathrm{km}^{2}\right)\end{array}$ & $\begin{array}{c}\text { Densidad Pob } \\
(6-12)\end{array}$ & $\begin{array}{c}\text { Densidad de } \\
\text { colegios } \\
\left(\text { colegio } / \mathrm{km}^{2}\right) \\
\end{array}$ \\
\hline Cabo de Gata-Nijar & 3 & 500 & 232222 & 16749 & 66 & 464,44 & 33,50 & 0,13 \\
\hline Sierras Subbéticas & 8 & 320 & 68537 & 3927 & 23 & 214,18 & 12,27 & 0,07 \\
\hline Sobrarbe-Pirineos & 19 & 2202 & 7317 & 368 & 3 & 3,32 & 0,17 & 0,00 \\
\hline Costa Vasca & 3 & 89 & 20856 & 1425 & 6 & 234,34 & 16,01 & 0,07 \\
\hline Sierra Norte de Sevilla & 10 & 1774,84 & 25583 & 1468 & 12 & 14,41 & 0,83 & 0,01 \\
\hline Villuercas Ibores Jara & 19 & 2544,4 & 13254 & 456 & 8 & 5,21 & 0,18 & 0,003 \\
\hline Cataluña Central & 37 & 1300 & 190628 & 13076 & 55 & 146,64 & 10,06 & 0,04 \\
\hline Molina-Alto Tajo & 77 & 4520 & 9253 & 319 & 4 & 2,05 & 0,07 & 0,00 \\
\hline El Hierro & 3 & 595 & 10679 & 526 & 8 & 17,95 & 0,88 & 0,01 \\
\hline Lanzarote y Archipiélago Chinijo & 7 & 2500 & 147023 & 9830 & 42 & 58,81 & 3,93 & 0,02 \\
\hline Las Loras & 16 & 950,76 & 12200 & 444 & 5 & 12,83 & 0,47 & 0,01 \\
\hline Conca de Tremp-Montsec & 19 & 2050 & 16029 & 820 & 12 & 7,82 & 0,40 & 0,01 \\
\hline TOTAL & 221 & 19346 & 753581 & 49408 & 244 & 38,95 & 2,55 & 0,01 \\
\hline
\end{tabular}

TABLA N ${ }^{\circ}$ 3. Características geográficas de los geoparques españoles (año 2018)

Fuente: elaborado a partir del INE y de la información proporcionada por las Consejerías de Educación de las Comunidades Autónomas que disponen de geoparque.

De forma más precisa, si se centra la atención en el número de habitantes en edad de estudiar EP que pueblan los municipios que integran los geoparques españoles, nos encontramos que esta figura afecta directamente a un total de 49.408 habitantes. Con estos datos, podríamos resaltar que la densidad de población para este tipo de habitantes es de 2,55 estudiantes de EP/ $\mathrm{km}^{2}$. En este sentido, las densidades entre unos espacios y otros también se encuentran notablemente desequilibradas. Se trata de un número reducido de estudiantes de primaria los que pueden ser recibidos de forma directa por los geoparques, situación, a la postre, que podría considerarse como ventajosa al poder disfrutar de un espacio con notables cualidades didácticas que en el momento actual no se encuentra masificado.

De forma directa, el total de colegios en los que se oferta EP y que se encuentran bajo el radio territorial de acción de los geoparques es de 244. Por el momento, existe la

5 Para la realización de esta tabla no se han tenido en consideración los datos relativos al Geoparque do Courel debido a su reciente incorporación a la red global de la UNESCO durante el año 2019 y no se encuentra disponible su proyecto educativo. 
posibilidad de crear un tejido escolar intraparque e interparque que agrupe a un amplio número de centros educativos que, cumpliendo las premisas que emite la UNESCO en cuanto a colaboración intercentros, se encuentran en disposición de realizar intercambios, tanto de experiencias como de materiales didácticos.

\section{LOS GEOPARQUES COMORECURSODIDÁCTICOPARALAENSEÑANZA DEL PAISAJE DE MONTAÑA EN EDUCACIÓN PRIMARIA: PROPUESTAS DE LOS GEOPARQUES ESPAÑOLES.}

Uno de los requisitos previos que han de cumplir los territorios que pretenden ser catalogados con la figura geoparque es el de acometer el desarrollo de un proyecto educativo dirigido a todas las edades, tanto en el ámbito de la enseñanza formal como en el de la no formal (UNESCO, 2018).

En el caso que nos ocupa, los geoparques españoles cuentan con un proyecto educativo sólido, orientado a la formación y a la educación en materia ambiental, paisajística, social, cultural y, por supuesto, geológica y geomorfológica. En este sentido, nos encontramos ante una figura que trata de poner en valor el trabajo in situ, la excursión, como mecanismo para afrontar el proceso de enseñanza-aprendizaje. Aunque disponen de actividades y materiales que cubren todos los rangos de edad, en el presente epígrafe serán los orientados a la etapa de EP los que centrarán nuestra atención. Para ello, se realizará una catalogación y un análisis comparativo de las propuestas comunes a todos los geoparques con el objeto de valorizar la oferta realizada.

Todos ellos cuentan con dos tipos de planteamientos, por un lado ponen en marcha aquellas actividades que están orientadas al público en general (educación no formal) que pueden ser realizadas por los estudiantes de EP en el ámbito familiar. Por el otro, presentan un conjunto de acciones formativas enmarcadas en los contextos formales de la educación en colaboración con los centros educativos de sus áreas de acción.

En cuanto a las propuestas ofertadas para el ámbito no formal, la gran mayoría se centran en el desarrollo de rutas, bien guiadas por un experto del geoparque, bien regidas por hitos y cartelería para ser realizadas de forma autónoma. Ambos casos se encuentran complementados por las visitas a los centros de interpretación y de recepción con los que cuentan estos espacios. Los recorridos transcurren uniendo diferentes geositios donde los visitantes pueden analizar los diversos elementos naturales que articulan los paisajes de montaña (orografía, geología, hidrología, vegetación, etc.) Del mismo modo, de forma periódica se ofrece la posibilidad de realizar talleres formativos en materia ambiental, paisajística y cultural.

Es en el contexto de la educación formal donde se define una diversificada oferta, tanto de materiales como de actividades didácticas, en colaboración con los centros 
educativos. En este sentido, las propuestas centradas en el paisaje de montaña adquieren mayor relevancia. No se ha de pasar por alto que todas ellas van a estar definidas por las peculiaridades territoriales y culturales del área en cuestión, aunque los contenidos trabajados sean los mismos en todos los geoparques.

Gran parte de las actividades desarrolladas desde los geoparques están centradas, para la etapa de Primaria, en los contenidos relativos al paisaje (paisaje y sus elementos, paisaje de montaña, acción humana sobre el paisaje, evolución paisajística), a la geología y a la conservación y concienciación ambiental.

Igualmente, la cooperación con los centros educativos favorece la elaboración de unidades didácticas articuladas para el trabajo, tanto en el aula como en los espacios de los geoparques. Aunque, en principio, estas unidades están diseñadas por los técnicos de estos espacios es frecuente la contribución de los profesionales docentes para, de este modo, adaptarse a las particularidades del centro y a las necesidades del aula y alumnado.

A grandes rasgos, todos los que integran la red española ofrecen los siguientes elementos o actividades: material didáctico, actividades y talleres, cursos formativos, jornadas de geoconvivencia y rutas diseñadas para los centros educativos. Además del abanico de actividades comunes existe una diversificada oferta de propuestas educativas de carácter cooperativo que integran, bien a varios geoparques en proyectos comunes, bien a un conjunto de colegios en el proyecto de un geoparque. En este sentido se destacan a continuación de forma muy somera algunos de los ejemplos que han sido analizados.

En el caso concreto de los geoparques andaluces (Cabo de Gata-Nijar, Sierra Norte de Sevilla y Sierras Subbéticas) a través de los programas de la Junta de Andalucía " $L a$ Naturaleza y Tû" y "Naturaleza menuda", los centros educativos cuentan con una amplia oferta de actividades centradas en los contenidos relativos a los elementos naturales del paisaje. Se trata de dos proyectos de índole autonómica que ponen a disposición de los centros educativos toda la oferta de actividades que pueden ser realizadas en los geoparques y en los espacios naturales protegidos de la red andaluza.

El Geoparque Sobrarbe-Pirineos ha llevado a cabo durante los últimos cursos académicos una serie de talleres centrados en la cultura agro-pastoril, en el marco del programa "Estudio y difusión del pastoralismo en el bien Pirineos Monte Perdido II" centrado en la construcción del patrimonio cultural y paisajístico. En ellos se han puesto en marcha actividades sobre la trashumancia, la prehistoria, la acción antrópica sobre el paisaje, etc.

${ }^{6}$ Programas configurados para ser desarrollados, además de en los geoparques, en los espacios naturales protegidos de la Comunidad Autónoma a través de la siguiente página web: www. reservatuvisita.es 


\section{Rubén Fernández Álvarez}

El Geoparque Costa Vasca en la actualidad está inmerso en un proyecto de ámbito internacional que trabaja por el desarrollo de una ruta educativa y geoturística Atlánticaeuropea que recibe la denominación de Atlantics Geoparks. Consiste en la unión, a través de un programa educativo común, de aquellos geoparques que se encuentran ubicados en el Arco Atlántico con el objetivo de impulsar propuestas de atracción turística centradas en la educación y en la ciencia.

Por su parte, el Geoparque Villuercas Ibores Jara a través de proyecto educativo Geocentros ha puesto en marcha un proceso colaborativo intercentros para desarrollar una propuesta didáctica en cada uno de los colegios que pueda ser utilizada por el resto de centros integrantes. En este sentido, se están elaborando una serie de murales que actúan de guión para el trabajo de algunos de los contenidos fijados para la EP. Entre el material didáctico que pone a disposición de los colegios es de resaltar la geomaleta. Consiste en una colección de minerales, rocas y fósiles presentes en el geoparque así como una serie de fichas descriptivas que contribuyen al buen desarrollo del proceso de enseñanza-aprendizaje.

Los geoparques han de trabajar por una participación activa de los centros educativos fomentando la creación de redes de intercambio de conocimiento entre estos espacios y los colegios y entre los propios colegios del entorno, surgiendo así un variado "banco" de materiales y métodos a disposición de todos los docentes. Así, se ha construido un tejido de guías y unidades didácticas que pueden ser utilizadas por los docentes para poner en marcha el proceso de enseñanza-aprendizaje centrado en el paisaje, más concretamente en el paisaje de montaña, pues todos estos espacios están caracterizados por su localización en áreas de montaña.

\section{DISCUSIÓN Y CONCLUSIONES}

Los geoparques son un instrumento de cualidades ambientales que desarrollan dos líneas complementarias de acción: geoturismo y geoeducación. Con la primera de ellas tratan de impulsar nuevas acciones socioeconómicas que repercutan en el desarrollo del territorio. Con su segunda línea, orientada hacia el ámbito de la enseñanza, se centran en el desarrollo de un plan educativo en el que la concienciación ambiental y el conocimiento de los valores naturales y culturales del espacio geográfico sean sus dos pilares fundamentales. Trabajos como los de Azman et al. (2010) y Farsani et al. (2017) afirman que el uso de los geoparques como recurso didáctico favorecen la integración de nuevos instrumentos en los procesos de enseñanza-aprendizaje de los elementos territoriales y del paisaje y, además, contribuye a la creación de conciencia ambiental en la sociedad. Siguiendo estos mismos postulados, Fernández (2018) considera que a través de sus proyectos educativos los geoparques ponen a disposición de la ciudadanía 
una serie de recursos y de actividades que pueden utilizarse y llevarse a cabo desde una triple vertiente: contexto escolar de aula; contexto escolar en el geoparque; aprendizaje autónomo en el marco familiar.

En esta línea los geoparques españoles cuentan con una serie de propuestas didácticas en las que el eje principal es el medio ambiente y la acción antrópica sobre el paisaje. Estas propuestas pueden ser utilizadas para la enseñanza de los contenidos paisajísticos que forman parte de los currículos autonómicos. Asimismo, estos persiguen el desarrollo de redes de colaboración educativa, tanto con la sociedad de su área de acción como con otros espacios que poseen la misma calificación. En este sentido Bitschene y Schüller (2011) consideran que los geoparques son una notable figura que facilita la cooperación educativa entre los colegios de un mismo geoparque y entre los diferentes geoparques que integran la red global. Además de la cooperación centro-espacio natural se fomenta la participación y el intercambio educativo entre los propios colegios, ya sea a nivel nacional o internacional.

Los proyectos educativos diseñados por los geoparques españoles promueven el trabajo autónomo e inclusivo de los discentes, la transversalidad de contenidos y el aprendizaje de tipo significativo. Siguiendo esta tendencia, los trabajos realizados por Catana y Rocha (2009) y por Rodrigues y Neto (2009) sobre el geoparque de Naturtejo expresan que la dinámica didáctica del proyecto educativo de este se centra en la transversalidad y en la alternancia de trabajo autónomo y trabajo cooperativo entre los estudiantes. Esta apuesta docente se ciñe perfectamente a las propuestas que han desarrollado algunos autores que centran su trabajo en la didáctica del paisaje. Así, Bajo (2001), Batllorí y Serra (2017) y Busquets (2011) coinciden al afirmar que el paisaje es un notable recurso que facilita la enseñanza de tipo transversal y el contacto con el espacio geográfico. Igualmente, a este respecto se ha de destacar que las actividades propuestas por los geoparques se encuentran diseñadas para su desarrollo en el campo a través de excursiones. Esta metodología de trabajo in situ es recomendada para la enseñanza del paisaje (Bajo, 2001; Liceras, 2003) pues favorece el aprendizaje de tipo significativo y la comprensión de las interrelaciones entre los elementos que lo integran.

Los geoparques localizados en el territorio estatal ofertan una extensa variedad de propuestas didácticas para la etapa de Educación Primaria que afectan directamente a un amplio número de centros educativos, pues todos los localizados en el área de acción de esta figura pueden participar en el diseño y ejecución del proyecto educativo. Aunque las colaboraciones colegio-geoparques se dan principalmente en el ámbito territorial del geoparque, cualquier colegio podría solicitar una cooperación.

Específicamente para la EP los geoparques se convierten en un notable recurso para desarrollar el proceso de enseñanza-aprendizaje de algunos de los contenidos que vienen definidos, tanto por el marco nacional como por los instrumentos autonómicos 
para su implantación curricular. Concretamente, los contenidos relacionados con el paisaje y, de forma específica, con el paisaje de las áreas de montaña pueden ser tratados desde estos espacios pues ofertan una serie de propuestas que así lo facilitan y son llevados a cabo en el contexto de las áreas de montaña, aspecto este que posibilita la incorporación de estos contenidos. Del mismo modo, la proximidad con los elementos que integran el paisaje (vivencias sobre los mismos) puede ayudar al desarrollo de valores de concienciación y conservación en materia ambiental (Azman et al. 2010; Farsani et al. 2017).

Nos encontramos ante un notable recurso didáctico en el que el trabajo de campo y el contacto con el medio, aunados con el desarrollo de actividades educativas, individualizan y otorgan entidad a estos espacios frente a otras figuras de carácter natural en las que la vertiente proteccionista, emanada desde la normativa, son su elemento singularizador y no la elaboración de propuestas didácticas.

\section{BIBLIOGRAFÍA}

Azman, N., Halim, S.A., Liu, O.P., Saidin, S., Komoo, I. (2010). Public education in heritage conservation for Geopark community. En Jelas, Z.M., Salleh, A., Azman, N. (eds.) International Conference on Learner Diversity. Malaysa, ICED 2010, 504-511.

Bajo, M.J. (2001). El paisaje en el curriculum de educación primaria, dentro del área de conocimiento del medio natural, social y cultural. Revista de Pedagogía de la Universidad de Salamanca, (13), 51-61.

Batllori, R. y Serra, J. M. (2017). D'ensenyar geografia a través del paisatge a educar en paisatge. Documents d'Anàlisi Geogràfica, 63(3), 617-630.

Bitschene, P.; Schüller, A. (2011). Geo-education and geopark implementation in the Vulkaineifel European Geopark. In S. Carena, A.M. Friedrich, B. Lammerer (eds.) Geological Field Trips in Central Western Europe: Fragile Earth International Conference, (pp. 29-34). Munich: Geological Society of America Field Guide 22.

Bobrowsky, P.; Cronin, V.S.; Di Capua, G.; Kieffer, S.W.; Peppolini, S. (2017). The Emerging field of geoethics. In L.C. Gundersen (ed.), Scientific Integrity and Ethics with Applications to the Geosciences. Special Publication American Geophysical Union, John Wiley and Sons, Inc.

Busquets, J. (2010). La educación en paisaje: una oportunidad para la escuela. Íber, Didáctica de las Ciencias Sociales, Geografía e Historia, 65, 7-16.

Busquets, Jaume (2011). La importància de l'educació en paisatge. En: Nogué, J. et al. (eds.). Paisatge i educació. Olot, Observatori del Paisatge, 60-88. 
Busquets, J.; Cortina, A. (2016). Management of the territory: landscape management as a process. En Council Of Europe, Landscape Dimensions. Reflections and proposals for the implementation of the European Landscape Convention, Council of Europe Publishing, París, 25-54.

Calcagno, A. (2016). Landscape and Education. En Council of Europe, Landscape Dimensions. Reflections and proposals for the implementation of the European Landscape Convention, Council of Europe Publishing, París, 55-120.

Cardona, G. (2013). El "geocaching” y la didáctica de las Ciencias Sociales. Íber. Didáctica de las Ciencias Sociales, Geografía e Historia, 73, 26-34.

Casas, M. y Erneta, L. (2015). El paisaje en la Educación Secundaria Obligatoria. Una oportunidad educativa en el cambio curricular LOE-LOMCE. Didáctica Geográfica, 16, 45-71

Catana M.M.; Rocha, D. (2009). The role of the educational programs on tourism development of Naturtejo and Arouca geoparks. In: C. Neto de Carvalho and J. Rodrigues (ed.), Proceedings of 8th European Geoparks Conference, 14-16 September, Idanha-a-Nova, Portugal, 61-65

Consejo de Europa (2000): Convenio Europeo del Paisaje. Council of Europe, Cultural Heritage, Landscape and Spatial Planning Division, Florencia.

Dowling, R.K. (2013) Global Geotourism. An emerging form of sustainable tourism. Czech Journal of Tourism, 2, 59-79.

Eder, F.W.; Patzak, M. (2004). Geoparks-geological attractions: a tool for public education, recreation and sustainable economic development. Episodes, 27(3) 162-164.

EGN- European Geopark Network (2017). Geoparks. Recuperado de http://www. europeangeoparks.org/?page_id=6(Consultado27/12/2017)

Farsani, N.D., Coelho, C., Costa, C. (2011). Geotourism and Geoparks as novel strategies for socio-economic development in rural areas. International Journal of Tourism Researc, 13, 68-81.

Farsani, N.D.; Coelho, C.; Costa, C. (2013). Rural Geotourism: a new tourism product. Acta Geoturística, vol. 4 (2013), 2, 1-10.

Farsani, N.D.; Coelho, C.; Costa, C.; Amrikazemi,A. (2014). Geo-knowledge management and geoconservation via geoparks and Geotourism. Geoheritage, 6, 185-192

Farsani, N.D.; Mortazavi, M.; Bahrami, A.; Kalantary, R.; Bizhaem, F.K. (2017). Traditional Crafts: a Tool for geo-education in Geoturism. Geoheritage, 2017. DOI 10.1007/s12371-016-0211-2 
Fernández, R. (2015). La aplicación de Landscape Character Assessment a los espacios de montaña media: el paisaje del macizo de Las Villuercas. Ciudad y Territorio. Estudios Territoriales, 185, 499-518.

Fernández, R. (2016). El geoparque como figura turística y didáctica para el fomento del desarrollo endógeno. Red de Geoparques de España: análisis y caracterización. En Madureira, L., Silva, P.G., sacramento, O., Marta-Costa, A., Koehnen, T. Smart and Inclusive Development in Rural Areas. Book of proceedings of th 11 Iberian Conference on Rural Studies.

Fernández, R. (2018). El Geoparque como recurso didáctico: espacio geográfico, montaña y paisaje. In E. López, C.R. García and M. Sánchez (eds.), Buscando formas de enseñar: investigar para innovar en Didáctica de las Ciencias Sociales (pp. 883-893). Valladolid: Ediciones de la Universidad de Valladolid.

García de la Vega,A. (2012). Un enfoque innovador en la didáctica del paisaje: escenario y secuencia geográfica. En de Miguel, R., de Lázaro, M.L., Marrón, M.J. (coords.) La educación geográfica digital. Asociación de Geógrafos Españoles y Universidad de Zaragoza. Zaragoza, 455-470.

Gómez-Mendoza, J. (2013). Del patrimonio paisaje a los paisajes patrimonio. Documents d'Analisi Geogràfica, 59 (1), 5-20.

González-Tejeda, C.; Du, Y.; Read, M. Girault, Y. (2017). From nature conservation to Geotourism development: Examining ambivalent attitudes towards UNESCO directives with the global geopark network. International Journal of Geoheritage, $5(2), 1-20$.

Granados, J. (2011). La educación para la sostenibilidad en la enseñanza de la Geografía. Un studio de caso. Enseñanza de las Ciencias Sociales, 10, 31-43.

Hernández, A.M., Jaraiz, F.J., Gurría, J.L. (2015). Aprender en y con el paisaje cultural: Las Hurdes (Extremadura). Íber. Didáctica de las Ciencias Sociales, Geografía e Historia, 81, 22-28.

Hernández, F.X., Rivero, M.P. (2015). Aprender con y a través del paisaje cultural. Íber. Didáctica de las Ciencias Sociales, Geografía e Historia, 81, 1-3.

Hernández, M. (2009). El paisaje como seña de identidad territorial: valorización social y factor de desarrollo, ¿utopía o realidad? Boletín de la Asociación de Geógrafos Españoles, 49, 169-183.

Hoepfl, M.C. (1997). Choosing Qualitative Research: A primer for technology education researchers. Journal of Technology Education, vol. 9(1), 47-63

Hose, T.A. (2012). 3G's for modern geotourism. Geoheritage, 4, 7-24. 
Justice, S.C. (2018). UNESCO Global geoparks, Geotourism and Communication of the Earth Sciences: A case study in the Chablais UNESCO Global Geopark, France. Geosciences, 8, 149. doi:10.3390/geosciences8050149

Lewis, G.M.; Hampton, S.J. (2015). Visualizing volcanic processes in SketchUp: An integrated geo-education tool. Computers and Geosciences, 81, 93-100.

Liceras, A. (2003). Observar e interpretar el paisaje: Estrategias didácticas. Granada, Grupo Editorial Universitario.

Liceras, A. (2013). Didáctica del paisaje. Íber. Didáctica de las Ciencias Sociales, Geografía e Historia, 74, 85-93.

López, J. (2016). Los Geoparques mundiales UNESCO como estrategias de desarrollo territorial. En Leco, F. (coord.) Territorio y desarrollo rural: aportaciones desde el ámbito investigador. Cáceres, Junta de Extremadura, 177-194.

Martínez, R y Arrebola, J.C. (2016). La enseñanza del paisaje en España. Una mirada a través de los manuales escolares de Ciencias Sociales. Revista Contexto \& Educação, 31(99), 9-33.

Massot, I.; Dorio, I.; Sabariego, M. (2004). Estrategias de recogida y análisis de la información. In Metodología de la investigación educativa, Bisquerra, R., Ed.; Editorial La Muralla: Madrid, Spain, pp. 321-358

McKeever, P.J.; Zouros, N. (2005). Geoparks: celebrating Earth heritage, sustaining local communities. Episodes, vol. 28(4), 274-278.

Mieczkowski, Z. (1995). Environmental issues of tourism and recreation. New York: University Press of America.

Mikhailenko, A.V.; Ruban, D.A. (2019). Geo-Heritage specific visibility as an important parameter in geo-tourism resource evaluation. Geosciences, 9, 146, doi:10.3390/ geosciences 9040146

Miralbés, R.; Higueras, A. (1993). Reflexiones sobre el Espacio Geográfico. Geographicalia, 30: 283-294.

Nardi, A. (2010). El paisaje como instrumento de intermediación cultural en la escuela. Íber, Didáctica de las Ciencias Sociales, Geografía e Historia, 65: 27-34.

Nogué, J.; Puigbert, L.; Sala, P.; Bretcha, G. (2010): Landscape and Public Participation. The Experience of the Landscape Catalogues of Catalonia. Observatorio del Paisaje de Cataluña y Generalitat de Catalunya, Barcelona. 
Newsome, D.; Dowling, R.K. (2010). Setting an-agenda for geotourism. In D. Newsome, R. Dowling, R. (Eds.), Geotourism: The Tourism of Geology and Landscape (pp. 1-22). Oxford: Goodfellow Publishers Limited.

Ngwira,P.M. (2015). Geotourism and geoparks: Africa's current prospects for sustainable rural development and poverty alleviation. In E. Errami, M. Brocx, V. Semeniuk (eds.). From geoheritage to geoparks. Case studies from Africa and beyond, (pp. 25-33). New York: Springer.

Ólafsdóttir, R.; Tverijonaite, E. (2018). Geotourism: a systematic literatura review. Geosciences, 8, 234, 1-16. http://dx.doi.org/10.3390/geosciences8070234

Ólasfsdóttir, R., Dowling, R. (2014). Geotourism and Geoparks. A Tool for Geoconservation and Rural Develoopment in Vulnerable Environments: A case study from Iceland. Geoheritage, 6, 61-77.

Ortega, M. (2007): El Convenio Europeo del Paisaje: Claves para un compromiso. Ambienta, $\mathrm{n}^{\circ}$ 63: 18-26.

Pérez, R.; Galán, A.; Quintanal, J. (2012). Métodos y diseños de investigación en educación. Universidad Nacional de Educación a Distancia: Madrid, Spain.

Pforr, C.; Megerle, A. (2006). Geotourism: a perspective from southwest Germany. In R.K. Dowling, D. Newsome (eds.) Geotourism: sustainability, impacts and management, (118-139). Oxford: Elsevier Butterworth-Heinemann.

Prats, J.; Busquets, J. (2010). Didáctica del paisaje. Íber, Didáctica de las Ciencias Sociales, Geografía e Historia, 65: 5-6.

Rodrigues, J.C.; Neto, C. (2009). Geoproducts in geopark Naturtejo, Proceedings of 8th European Geoparks Conference, Idanha-a-Nova, Portugal, 82-86.

Tejada, L. (2009). Las salidas, un recurso para el aprendizaje en Educación Infantil. Innovación y experiencias educativas. Revista digital, 14, 1-11.

Thomashow, M. (1995). Ecological Identity, becoming a reflective environmentalist. London. MIT Pres.

Torres-Porras, J., Alcántara, J., Arrebola, J.C., Rubio, S.J. y Mora, M. (2017). Trabajando el acercamiento a la naturaleza de los niños y niñas en el Grado de Educación Infantil. Crucial en la sociedad actual. Revista Eureka sobre Enseñanza y Divulgación de las Ciencias, 14 (1), 258-270.

UNESCO (2017). Earth Sciences. Recuperado de http://www.unesco.org/new/en/ natural-sciences/environment/earth-sciences/unesco-global-geoparks/ (Consultado 23/12/2017). 
UNESCO (2018). UNESCO Global Geoparks and Education. Recuperado de http:// www.unesco.org/new/en/natural-sciences/environment/earth-sciences/unescoglobal-geoparks/top-10-focus-areas/education/ (Consultado 10/05/2018).

UNESCO (2019). Earth Science for Society. In UNESCO. Recuperado de http://www. unesco.org/new/en/natural-sciences/environment/earth-sciences/unesco-globalgeoparks/

Wojtowicz, B.; Wojtowicz, P. (2016). The prospects for the development of Geotourism in the Area of the Wadi Rum Desert. Journal of Tourism and Hospitality Management, vol. 4(1), 1-14.

Zoido, F. (2000). El paisaje, ideas para la actuación. En Martínez de Pisón (dir.) Estudios sobre el paisaje. Fundación Duques de Soria y Ediciones de la Universidad Complutense de Madrid, Madrid: 293-311.

Zoido, F. (2004): El paisaje, patrimonio público y recurso para la mejora de la democracia. Boletín del Instituto Andaluz del Patrimonio Histórico, 50: 66-73.

Zoido, F. (2006a). Landscape and spatial planning policies. En Council of Europe. Lanscape and sustainable development: challenges of European Landscape Convention. Council of Europe Publishing, Strasbourg: 55-82.

Zoido, F. (2006b). «Principales retos de adaptación de la Convención Europea del Paisaje a las políticas de ordenación del territorio en Europa». En Mata y Torroja (coords.), El paisaje y la gestión del territorio. Criterios paisajísticos en la ordenación del territorio y el urbanismo. Diputación de Barcelona-Universidad Internacional Menéndez Pelayo, Barcelona: 359-374.

Zouros, N. (2004). The European Geoparks Network. Episodes, vol. 27 (3), 165-171.

Zouros, N. (2010). Lesvos Petrified Forest Geopark, Greece: Geotourism and Local Development. The George Wright Forum Journal, 27 (1), 19-28.

Zouros, N. y Martini, G. (2003). Introduction to the European geoparks network, In NHM of Lesvos Petrified Forest. Proceedings of the 2nd International Symposium of Natural Monuments and Geological Heritage, Lesvos, 17-21. 
